CERN-TH/99-387

Cavendish-HEP-99/16

\title{
Fragmentation and Hadronization
}

\author{
B.R. Webber \\ Theory Division, CERN, 1211 Geneva 23, Switzerland, and \\ Cavendish Laboratory, University of Cambridge, Cambridge CB3 0HE, U.K.]
}

\section{Introduction}

Hadronic jets are amongst the most striking phenomena in high-energy physics, and their importance is sure to persist as searching for new physics at hadron colliders becomes the main activity in our field. Signatures involving jets almost always have the largest cross sections but are the most difficult to interpret and to distinguish from background. Insight into the properties of jets is therefore doubly valuable: both as a test of our understanding of strong interaction dynamics and as a tool for extracting new physics signals in multi-jet channels.

In the present talk I shall concentrate on jet fragmentation and hadronization, the topic of jet production having been covered admirably elsewhere [1, 2]. The terms fragmentation and hadronization are often used interchangeably, but I shall interpret the former strictly as referring to inclusive hadron spectra, for which factorization 'theorems'? are available. These allow predictions to be made without any detailed assumptions concerning hadron formation. A brief review of the relevant theory is given in sect. 2 .

Hadronization, on the other hand, will be taken here to refer specifically to the mechanism by which quarks and gluons produced in hard processes form the hadrons that are observed in the final state. This is an intrinsically non-perturbative process, for which we only have models at present. The main models are reviewed in sect. 3 . In sect. 1 their predictions, together with other less model-dependent expectations, are compared with the latest data on single-particle yields and spectra.

One of the most important objectives of jet studies is to understand the differences between jets initiated by different types of partons, especially quarks versus gluons,

\footnotetext{
${ }^{1}$ Plenary talk at XIX International Symposium on Lepton and Photon Interactions at High Energies, Stanford University, August 1999.

${ }^{2}$ Permanent address.

${ }^{3}$ I put this term in inverted commas because proofs of factorization in fragmentation do not really extend beyond perturbation theory [3].
} 
which could also be valuable in new physics searches. The wealth of recent data on quark-gluon jet differences is discussed in sect. 5. Another goal is to compare jets produced in different processes such as $e^{+} e^{-}$annihilation and deep inelastic scattering (DIS). This is being done by the H1 and ZEUS collaborations at HERA; some of their results are discussed in sect. 6 .

A good understanding of heavy quark jets is especially important since these are expected to be copiously produced in new processes such as Higgs boson decay. Section 7 discusses some recent results on heavy quark fragmentation.

Finally, sects. 8 and 9 deal with correlation effects, first those of Bose-Einstein origin and then those expected in fully hadronic final states from $e^{+} e^{-} \rightarrow W^{+} W^{-}$. A summary of the main points is given in sect. 10.

Regrettably I have no space to discuss many other relevant and interesting topics, such as: fragmentation function parametrizations [4]; photon fragmentation function; polarization in hadronization; event shapes and power corrections [5]; fluctuations and intermittency; dynamical two-particle correlations; heavy quark production in jets $(g \rightarrow c \bar{c}, b \bar{b})$; transverse energy flow in deep inelastic scattering; underlying event in DIS and hadron-hadron collisions; identified particle production in DIS; tests of QCD coherence; jet profiles and substructure [1]. Apologies also to all those whose work I have omitted or mentioned only superficially.

In citing the latest experimental data (mostly still preliminary) I have relied on the reference system of the EPS-HEP99 conference in Tampere, Finland [6], since few experimental papers were explicitly submitted to LP99. In the case of the large collaborations, papers submitted to EPS-HEP99 and/or LP99 can usually be found easily via the collaboration web pages [7]-[14].

\section{Jet fragmentation - theory}

Let us start by recalling the basic factorization structure of the single-particle inclusive distribution, e.g. in $e^{+} e^{-} \rightarrow h X$ (fig. 四):

$$
\begin{aligned}
& F^{h}(x, s)=\sum_{i} \int_{x}^{1} \frac{d z}{z} C_{i}\left(z, \alpha_{\mathrm{S}}(s)\right) D_{i}^{h}(x / z, s) \\
& s=q^{2}, \quad x=2 p_{h} \cdot q / q^{2}=2 E_{h} / E_{c m}
\end{aligned}
$$

where $C_{i}$ are the coefficient functions for this particular process (including all selection cuts etc.) and $D_{i}^{h}$ is the universal fragmentation function for parton $i \rightarrow$ hadron $h$.

The fragmentation functions are not perturbatively calculable but their $s$-dependence (scaling violation) is given by the DGLAP equation:

$$
s \frac{\partial}{\partial s} D_{i}^{h}(x, s)=\sum_{j} \int_{x}^{1} \frac{d z}{z} P_{j i}\left(z, \alpha_{\mathrm{S}}(s)\right) D_{j}^{h}(x / z, s)
$$




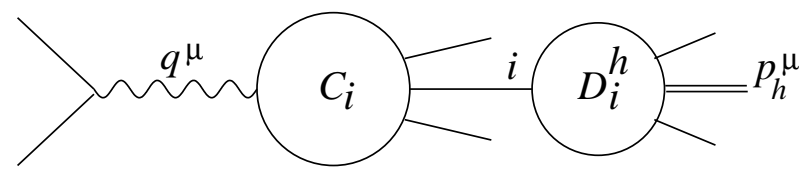

Figure 1: Factorization structure of $e^{+} e^{-} \rightarrow h X$.

Thus they can be parametrized at some fixed scale $s_{0}$ and then predicted at other energies [4.

In certain kinematic regions, higher-order corrections are enhanced by large logarithms, which need to be resummed. At small $x, \log x$ enhanced terms can be resummed by changing the DGLAP equation to

$$
s \frac{\partial}{\partial s} D_{i}^{h}(x, s)=\sum_{j} \int_{x}^{1} \frac{d z}{z} P_{j i}\left(z, \alpha_{\mathrm{S}}(s)\right) D_{j}^{h}\left(x / z, z^{2} s\right)
$$

This is commonly known as the modified leading-logarithmic approximation (MLLA) [15, 16, 17]. The effect of resummation is to generate a characteristic hump-backed shape in the variable $\xi=\ln (1 / x)$, with a peak at $\xi_{p} \sim \frac{1}{4} \ln s$.

Large logarithms of ratios of invariants may also appear inside the coefficient functions $C_{i}$, for example in three-jet events when the angles between jets become small. In some cases these can be absorbed into a change of scale in the fragmentation functions. Examples will be encountered in sects. 1 and 5.

Although universal, fragmentation functions are factorization scheme dependent. The splitting functions $P_{j i}$ are also scheme dependent in higher orders. To specify the scheme requires calculation of the coefficient functions to (at least) next-to-leading order. This has only been done in a few cases. Thus there is need for theoretical work to make full use of the data on fragmentation functions.

\section{Hadronization Models}

\subsection{General ideas}

Local parton-hadron duality [18]. Hadronization is long-distance process, involving only small momentum transfers. Hence the flows of energy-momentum and flavour quantum numbers at hadron level should follow those at parton level. Results on inclusive spectra and multiplicities support this hypothesis.

Universal low-scale $\alpha_{\mathrm{S}}$ [19, 20, 21]. Perturbation theory works well down to low scales, $Q \sim 1 \mathrm{GeV}$. Assume therefore that $\alpha_{\mathrm{S}}\left(Q^{2}\right)$ can be defined non-perturbatively for all $Q$, and use it in evaluation of Feynman graphs. This approach gives a good description of heavy quark spectra and event shapes. 


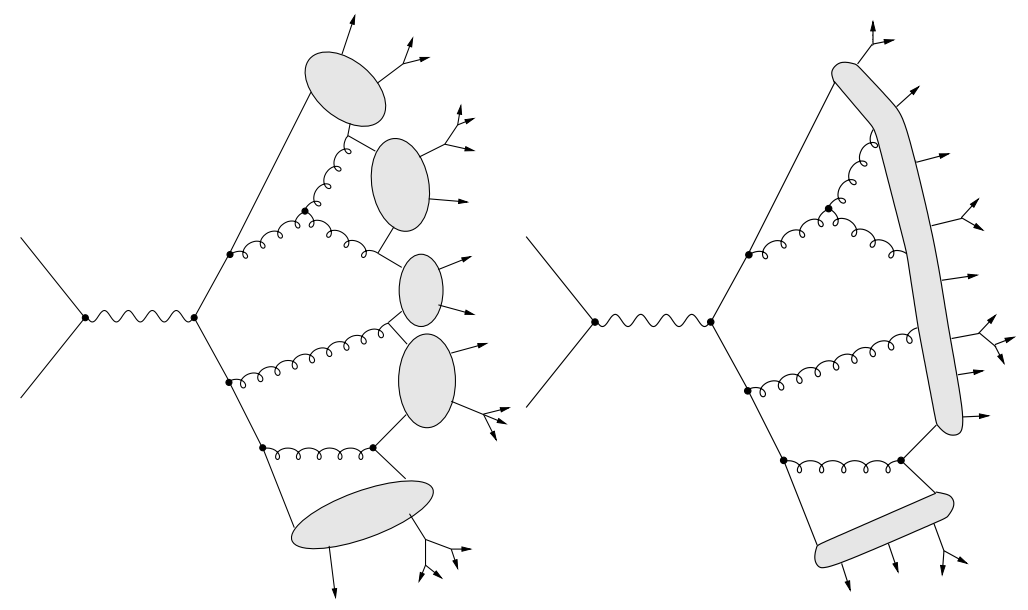

Figure 2: Cluster and string hadronization models.

\subsection{Specific models}

The above general ideas do not try to describe the mechanism of hadron formation. For this we must so far resort to models. The main current models are cluster and string hadronization. We describe briefly the versions used in the HERWIG and JETSET event generators, respectively.

- Cluster model [22]-[26]. The model starts by splitting gluons non-perturbatively, $g \rightarrow q \bar{q}$, after the parton shower. Colour-singlet $q \bar{q}$ combinations have lower masses and a universal spectrum due to the preconfinement [27, 28] property of the shower (fig. [3 [29]). These colour-singlet combinations are assumed to form clusters, which mostly undergo simple isotropic decay into pairs of hadrons, chosen according to the density of states with appropriate quantum numbers [23]. This model has few parameters and a natural mechanism for generating transverse momenta and suppressing heavy particle production in hadronization. However, it has problems in dealing with the decay of very massive clusters, and in adequately suppressing baryon and heavy quark production.

- String model [30]-334. This model is based on the dynamics of a relativistic string, representing the colour flux stretched between the initial $q \bar{q}$. The string produces a linear confinement potential and an area law for matrix elements:

$$
\left|M\left(q \bar{q} \rightarrow h_{1} \cdots h_{n}\right)\right|^{2} \propto e^{-b A}
$$

where $A$ is the space-time area swept out (fig. 四). The string breaks up into hadrons via $q \bar{q}$ pair production in its intense colour field. Gluons produced in the 


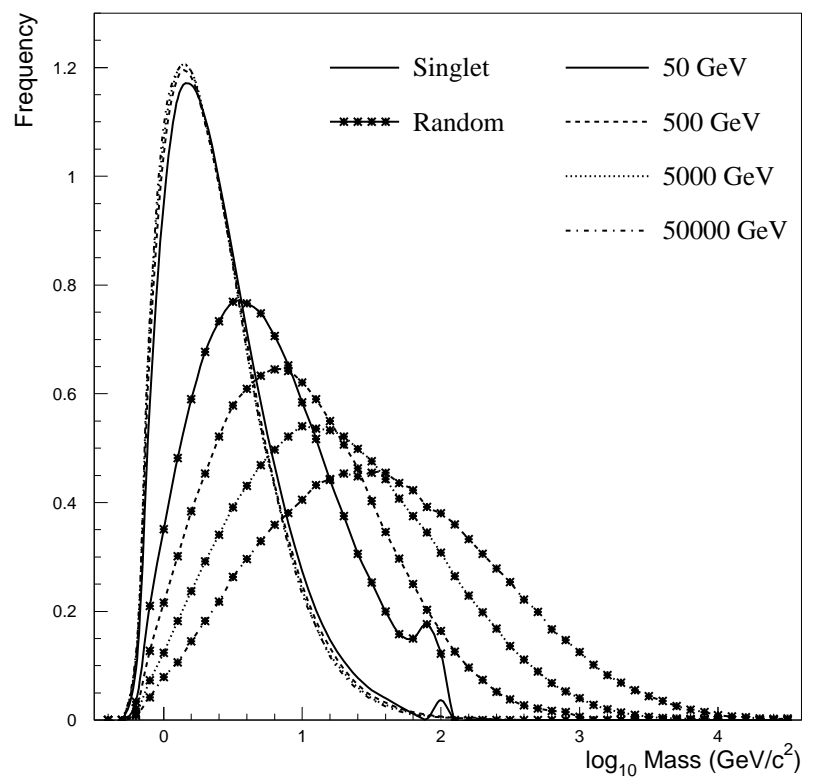

Figure 3: Cluster model: mass distribution of $q \bar{q}$ pairs.

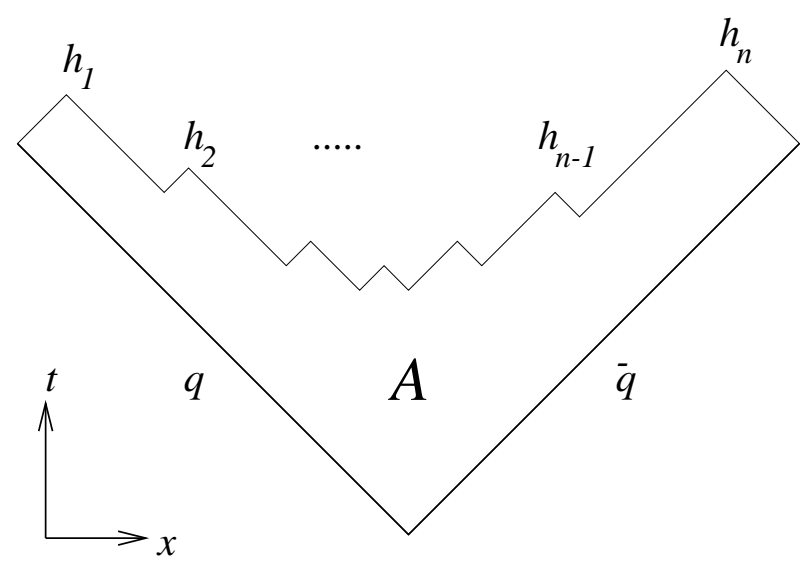

Figure 4: String model: space-time picture. 
parton shower give rise to 'kinks' on the string. The model has extra parameters for the transverse momentum distribution and heavy particle suppression. It has some problems describing baryon production, but less than the cluster model.

- The UCLA model [35]-[37] is a variant of the JETSET string model which takes the above area law for matrix elements more seriously, using it to determine the relative rates of production of different hadron species. This results in heavy particle suppression without extra parameters, the mass-squared of a hadron being proportional to its space-time area. At present the model still uses extra parameters for $p_{T}$ spectra, and again has some problems describing baryon production.

\section{Single-particle yields and spectra}

Tables 1 and 2 compare predictions of the above models with data on $\mathrm{Z}^{0}$ decay from LEP and SLC. Of course, the models have tunable parameters, but the overall agreement is encouraging. As stated earlier, the main problems are in the baryon sector, especially for HERWIG.

It is remarkable that most measured yields (except for the $0^{-}$mesons, which have special status as Goldstone bosons) lie on the family of curves

$$
\langle n\rangle=a(2 J+1) e^{-M / T}
$$

where $M$ is the mass and $T \simeq 100 \mathrm{MeV}$ (fig. 5 [39]). This suggests that mass, rather than quantum numbers, is the primary factor in determining production rates. Note that, surprisingly, the orbitally-excited $J=\frac{3}{2}$ baryon $\Lambda(1520)$ (not yet included in models) is produced almost as much as the unexcited $J=\frac{3}{2}$ baryon $\Sigma(1385)$ [40, 41].

At other energies, model predictions for identified particle yields are in broad agreement with $e^{+} e^{-}$data (fig. 6 [42]), but statistics are of course poorer. Charged particle spectra at low $x$ agree well with the resummed (MLLA) predictions [15, 16, 17] over a wide energy range, as illustrated in fig. 0 [43].

In $p \bar{p} \rightarrow$ dijets [44] the relevant scale is taken to be $Q=M_{J J} \sin \theta$ where $M_{J J}$ is the dijet mass and $\theta$ is the jet cone angle (fig. 8). Results are then in striking agreement with theory and with data from $e^{+} e^{-}$annihilation at $Q=\sqrt{s}$ (fig. 9).

New SLD data include hadron spectra in light quark (rather than antiquark) fragmentation, selected by hemisphere using the SLC beam polarization [45]. One sees strong particle/antiparticle differences in the expected directions (fig. 10), bearing in mind the predominance of down-type quarks in $\mathrm{Z}^{0}$ decay.

\footnotetext{
${ }^{4}$ Updated from ref. [29].

${ }^{5}$ Recent ALEPH HERWIG tuning with strangeness suppression 0.8 [38].
} 


\begin{tabular}{|c|c|c|c|c|c|}
\hline Particle & Multiplicity & $\begin{array}{c}\text { HERWIG } \\
5.9\end{array}$ & $\begin{array}{c}\text { JETSET } \\
7.4\end{array}$ & $\begin{array}{c}\text { UCLA } \\
7.4\end{array}$ & Expts \\
\hline Charged & $20.96(18)$ & 20.95 & 20.95 & 20.88 & ADLMO \\
\hline$\pi^{ \pm}$ & $17.06(24)$ & 17.41 & 16.95 & 17.04 & $\mathrm{ADO}$ \\
\hline$\pi^{0}$ & $9.43(38)$ & 9.97 & 9.59 & 9.61 & ADLO \\
\hline$\eta$ & $0.99(4)$ & 1.02 & 1.00 & $\underline{0.78}$ & ALO \\
\hline$\rho(770)^{0}$ & $1.24(10)$ & 1.18 & 1.50 & $\overline{1.17}$ & $\mathrm{AD}$ \\
\hline$\omega(782)$ & $1.09(9)$ & 1.17 & 1.35 & 1.01 & ALO \\
\hline$\eta^{\prime}(958)$ & $0.159(26)$ & 0.097 & 0.155 & 0.121 & ALO \\
\hline$f_{0}(980)$ & $0.155(8)$ & $\underline{0.111}$ & $\sim \underline{0.1}$ & - & $\mathrm{ADO}$ \\
\hline $\mathrm{a}_{0}(980)^{ \pm}$ & $0.14(6)$ & 0.240 & - & 一 & $\mathrm{O}$ \\
\hline$\phi(1020)$ & $0.097(7)$ & 0.104 & $\underline{0.194}$ & $\underline{0.132}$ & $\mathrm{ADO}$ \\
\hline $\mathrm{f}_{2}(1270)$ & $0.188(14)$ & 0.186 & $\sim 0.2$ & - & $\mathrm{ADO}$ \\
\hline $\mathrm{f}_{2}^{\prime}(1525)$ & $0.012(6)$ & 0.021 & 二 & - & $\mathrm{D}$ \\
\hline $\mathrm{K}^{ \pm}$ & $2.26(6)$ & 2.16 & 2.30 & 2.24 & $\mathrm{ADO}$ \\
\hline $\mathrm{K}^{\mathbf{0}}$ & $2.074(14)$ & 2.05 & 2.07 & 2.06 & ADLO \\
\hline $\mathrm{K}^{*}(892)^{ \pm}$ & $0.718(44)$ & 0.670 & $\underline{1.10}$ & 0.779 & $\mathrm{ADO}$ \\
\hline $\mathrm{K}^{*}(892)^{0}$ & $0.759(32)$ & 0.676 & $\underline{1.10}$ & 0.760 & $\mathrm{ADO}$ \\
\hline $\mathrm{K}_{2}^{*}(1430)^{0}$ & $0.084(40)$ & 0.111 & - & - & $\mathrm{DO}$ \\
\hline $\mathrm{D}^{ \pm}$ & $0.187(14)$ & $\underline{0.276}$ & 0.174 & 0.196 & $\mathrm{ADO}$ \\
\hline $\mathrm{D}^{0}$ & $0.462(26)$ & $\overline{0.506}$ & 0.490 & 0.497 & $\mathrm{ADO}$ \\
\hline $\mathrm{D}^{*}(2010)^{ \pm}$ & $0.181(10)$ & 0.161 & $\underline{0.242}$ & $\underline{0.227}$ & $\mathrm{ADO}$ \\
\hline $\mathrm{D}_{\mathrm{s}}^{ \pm}$ & $0.131(20)$ & 0.115 & 0.129 & 0.130 & $\mathrm{O}$ \\
\hline $\mathrm{B}^{*}$ & $0.28(3)$ & 0.201 & 0.260 & 0.254 & $\overline{\mathrm{D}}$ \\
\hline $\mathrm{B}_{\mathrm{u}, \mathrm{d}}^{* *}$ & $0.118(24)$ & $\underline{0.013}$ & - & - & $\mathrm{D}$ \\
\hline $\mathrm{J} / \psi$ & $0.0054(4)$ & $\underline{0.0018}$ & 0.0050 & 0.0050 & ADLO \\
\hline$\psi(3685)$ & $0.0023(5)$ & 0.0009 & 0.0019 & 0.0019 & $\mathrm{DO}$ \\
\hline$\chi_{\mathrm{c} 1}$ & $0.0086(27)$ & $\underline{0.0001}$ & - & - & $\mathrm{DL}$ \\
\hline
\end{tabular}

Table 1: Meson yields in $\mathrm{Z}^{0}$ decay. Experiments: $\mathrm{A}=$ Aleph, $\mathrm{D}=$ Delphi, $\mathrm{L}=\mathrm{L} 3$, $\mathrm{M}=$ Mark II, $\mathrm{O}=$ Opal. Bold: new data this year. Underlined: disagreement with data by more than $3 \sigma$. 


\begin{tabular}{|c|c|c|c|c|c|}
\hline Particle & Multiplicity & $\begin{array}{c}\text { HERWIG } \\
5.9\end{array}$ & $\begin{array}{c}\text { JETSET } \\
7.4\end{array}$ & $\begin{array}{c}\text { UCLA } \\
7.4\end{array}$ & Expts \\
\hline $\mathrm{p}$ & $1.04(4)$ & $\underline{0.863}$ & $\underline{1.19}$ & 1.09 & ADO \\
\hline$\Delta^{++}$ & $0.079(15)$ & $\underline{0.156}$ & $\underline{0.189}$ & $\underline{0.139}$ & $\mathrm{D}$ \\
& $0.22(6)$ & 0.156 & 0.189 & 0.139 & $\mathrm{O}$ \\
\hline $\boldsymbol{\Lambda}$ & $0.399(8)$ & 0.387 & 0.385 & 0.382 & ADLO \\
$\boldsymbol{\Lambda}(\mathbf{1 5 2 0})$ & $0.0229(25)$ & - & - & - & DO \\
\hline$\Sigma^{ \pm}$ & $0.174(16)$ & 0.154 & 0.140 & 0.118 & DO \\
$\Sigma^{0}$ & $0.074(9)$ & 0.068 & 0.073 & 0.074 & ADO \\
$\Sigma^{\star \pm}$ & $0.0474(44)$ & $\underline{0.111}$ & $\underline{0.074}$ & $\underline{0.074}$ & ADO \\
\hline$\Xi^{-}$ & $0.0265(9)$ & $\underline{0.0493}$ & 0.0271 & $\underline{0.0220}$ & ADO \\
$\Xi(1530)^{0}$ & $0.0058(10)$ & $\underline{0.0205}$ & 0.0053 & 0.0081 & ADO \\
\hline$\Omega^{-}$ & $0.0012(2)$ & $\underline{0.0056}$ & 0.00072 & 0.0011 & ADO \\
\hline$\Lambda_{\mathrm{c}}^{+}$ & $0.078(17)$ & $\underline{0.0123}$ & 0.059 & $\underline{0.026}$ & O \\
\hline
\end{tabular}

Table 2: Baryon yields in $\mathrm{Z}^{0}$ decay. Legend as in table 1.

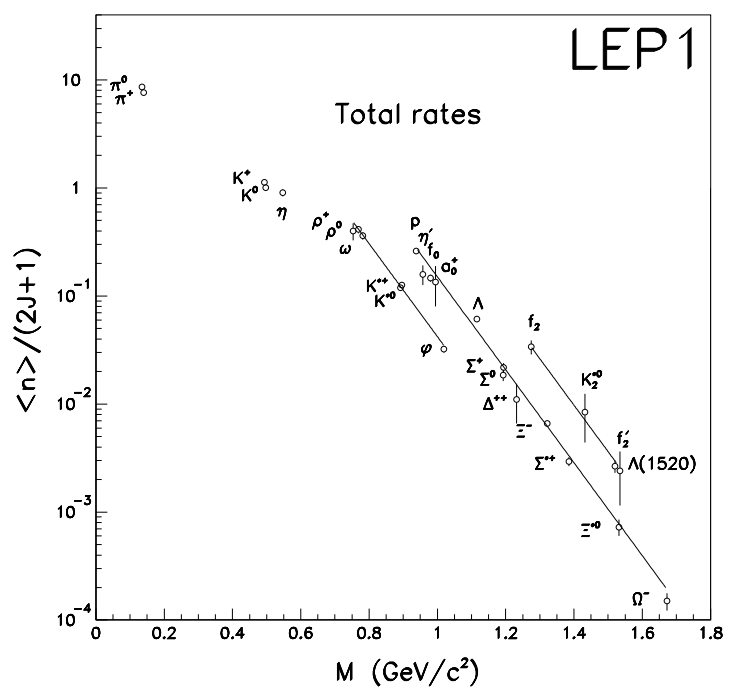

Figure 5: Particle yields in $\mathrm{Z}^{0}$ decay. 

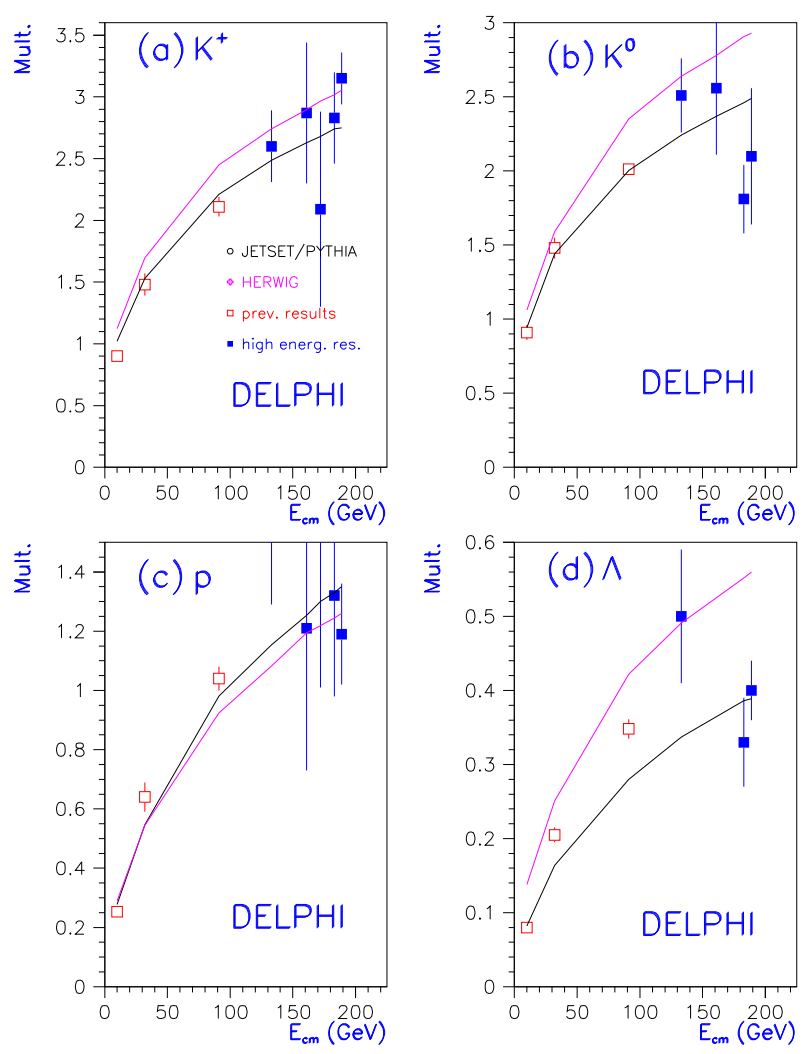

Figure 6: Particle yields in $e^{+} e^{-}$annihilation.
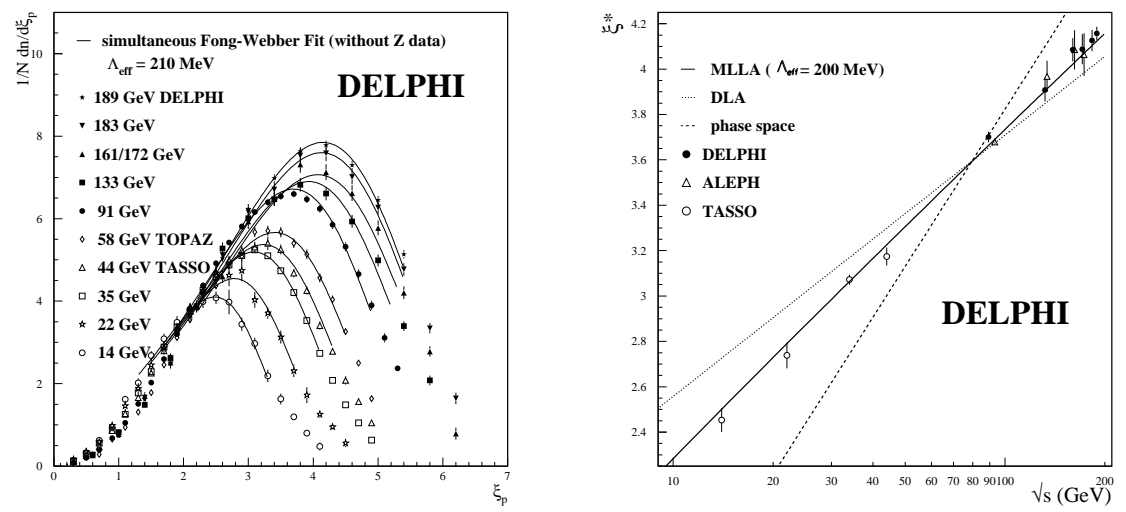

Figure 7: Low- $x$ fragmentation in $e^{+} e^{-}$annihilation. 


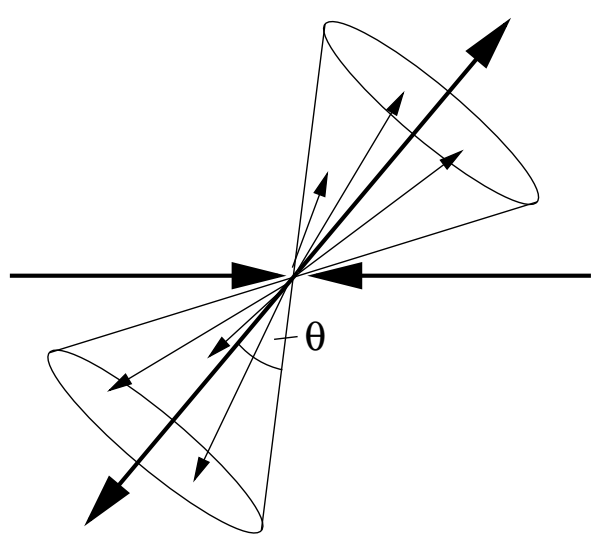

Figure 8: Cone angle in $p \bar{p} \rightarrow$ dijets
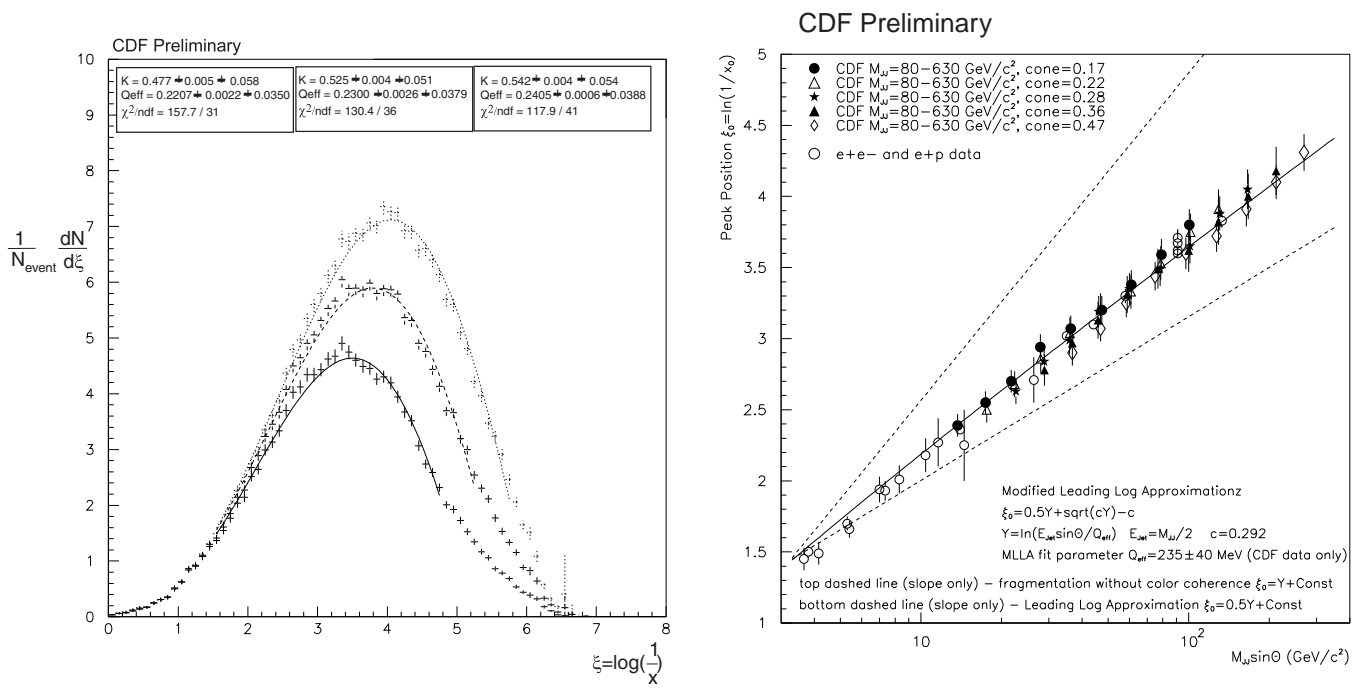

Figure 9: Low- $x$ fragmentation in $p \bar{p} \rightarrow$ dijets. 


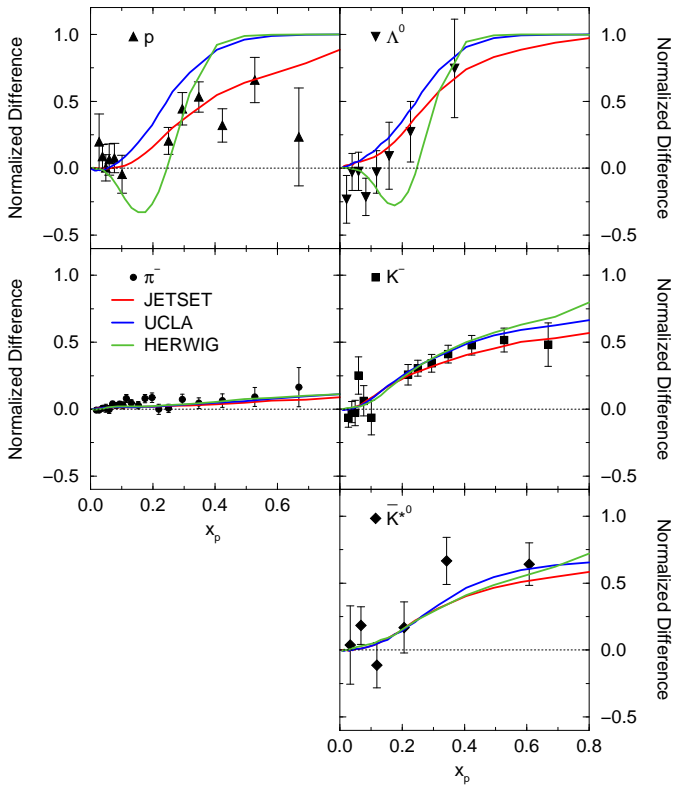

Figure 10: Normalized particle-antiparticle differences in quark jet fragmentation.

a)

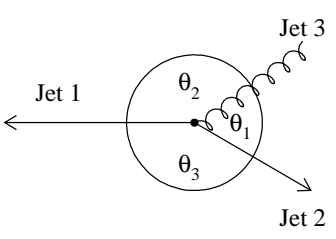

Y events b)

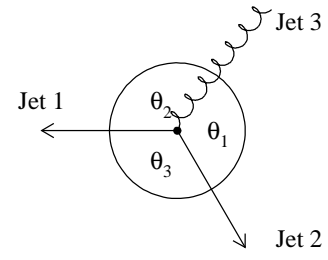

Mercedes events

Figure 11: Selection of gluon jets by DELPHI 


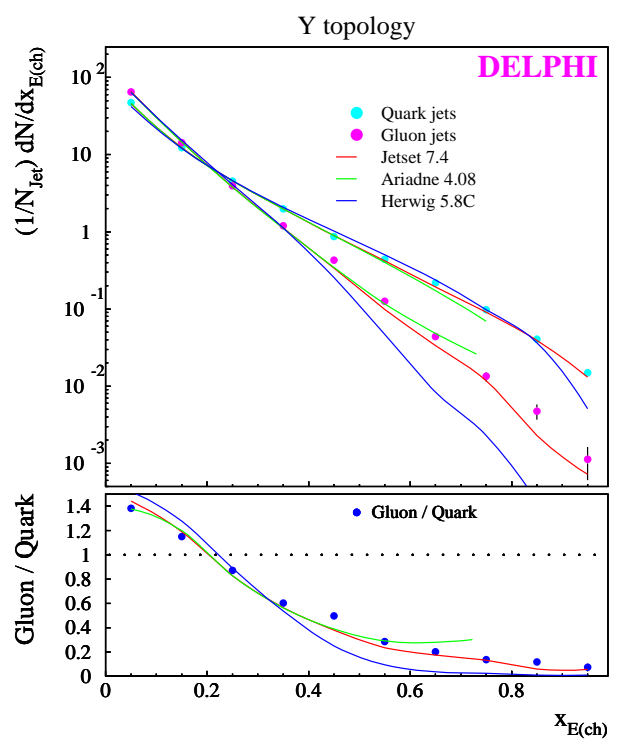

Figure 12: Charged particle spectra in quark and gluon jets.

\section{$5 \quad$ Quark and gluon jets}

DELPHI 46] select gluon jets by anti-tagging heavy quark jets in $\mathrm{Y}$ and Mercedes three-jet events (fig. 11). As expected, the higher colour charge of the gluon $\left(C_{A}=3\right.$ vs. $C_{F}=4 / 3$ ) leads to a softer spectrum and higher overall multiplicity (fig. 12). In general the relative multiplicities of identified particles are consistent with those of all charged, with no clear excess of any species in gluon jets (fig. 13). In particular there is no enhanced $\phi(1020)$ or $\eta$ production:

DELPHI [46]: $N_{g}(\phi) / N_{q}(\phi)=0.7 \pm 0.3$

OPAL [47]: $N_{g}(\eta) / N_{q}(\eta)=1.29 \pm 0.11$

OPAL 48] select gluon jets recoiling against two tagged $b$-jets in the same hemisphere. Monte Carlo studies indicate that such jets should be similar to those emitted by a point source of gluon pairs. The qualitative message from the data is again clear (fig. 14): Gluon jets have softer fragmentation than light quark jets, and higher multiplicity. The precision of the data is now such that next-to-leading order calculations of the relevant coefficient functions, taking into account the experimental selection 


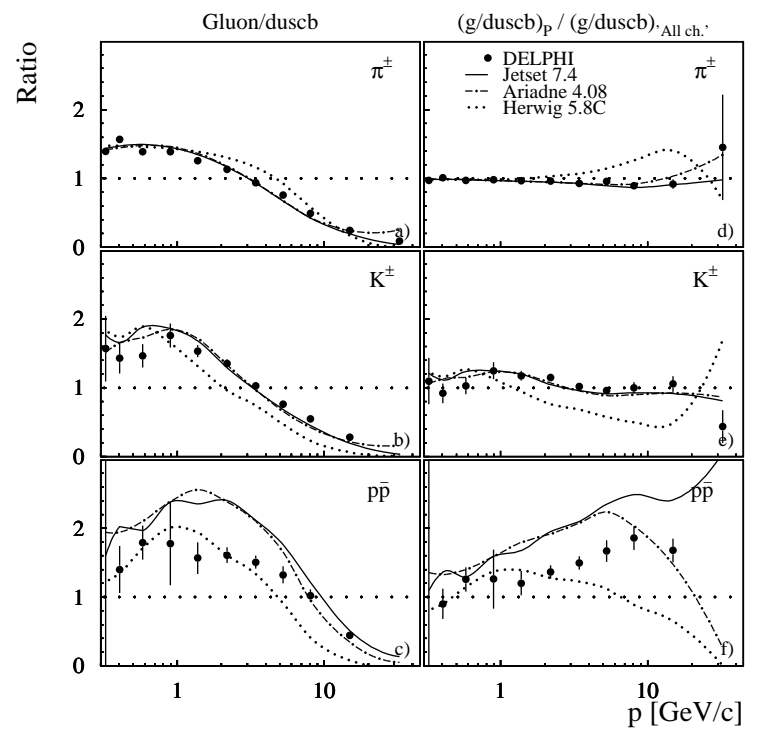

Figure 13: Comparisons of particle spectra in quark and gluon jets.

procedures, are needed to check universality of the extracted gluon fragmentation function.

The ratio of gluon/quark multiplicities at low rapidity (large angle) is close to the ratio of colour charges $r \equiv C_{A} / C_{F}=2.25$, in agreement with local parton-hadron duality:

$$
\text { OPAL: } r_{c h}(|y|<1)=1.919 \pm 0.047 \pm 0.095
$$

Monte Carlo studies 48] suggest that a better measure of $C_{A} / C_{F}$ is obtained by selecting low-momentum hadrons with relatively large $p_{T}$ (i.e. low rapidity). This gives

$$
\text { OPAL: } r_{c h}\left(p<4,0.8<p_{T}<3 \mathrm{GeV}\right)=2.29 \pm 0.09 \pm 0.015
$$

DELPHI 49] have observed scaling violation in quark and gluon jet fragmentation separately (fig. 15) by studying the dependence on the scale

$$
\kappa_{H}=E_{j e t} \sin (\theta / 2) \simeq \frac{1}{2} \sqrt{s y_{3}}
$$

where $\theta$ is the angle to the closest jet and $y_{3}$ is the Durham jet resolution 450 at which 3 jets are just resolved. This is expected to be the relevant scale when $y_{3}$ becomes small. One sees clearly that there is more scaling violation in gluon jets (fig. 16). The ratio provides another measure of $C_{A} / C_{F}$ :

$$
\text { DELPHI: } r_{\text {sc.viol. }}=2.23 \pm 0.09 \pm 0.06
$$



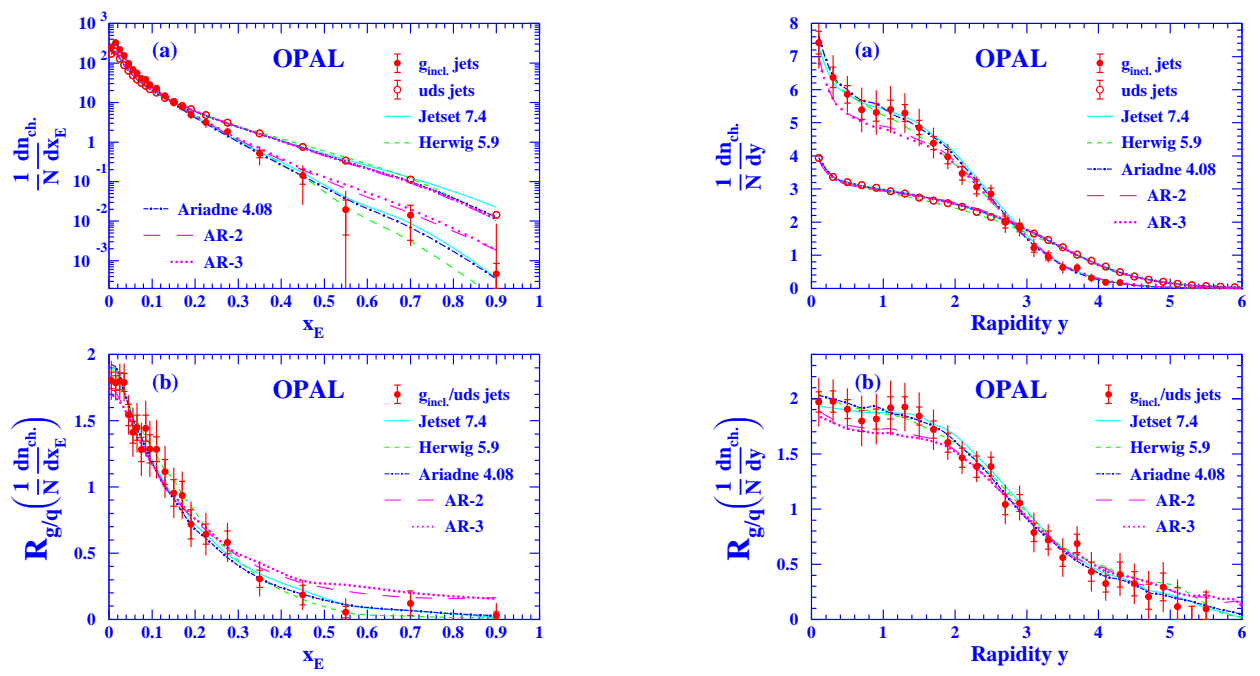

Figure 14: Momentum fraction and rapidity distributions in quark and gluon jets.
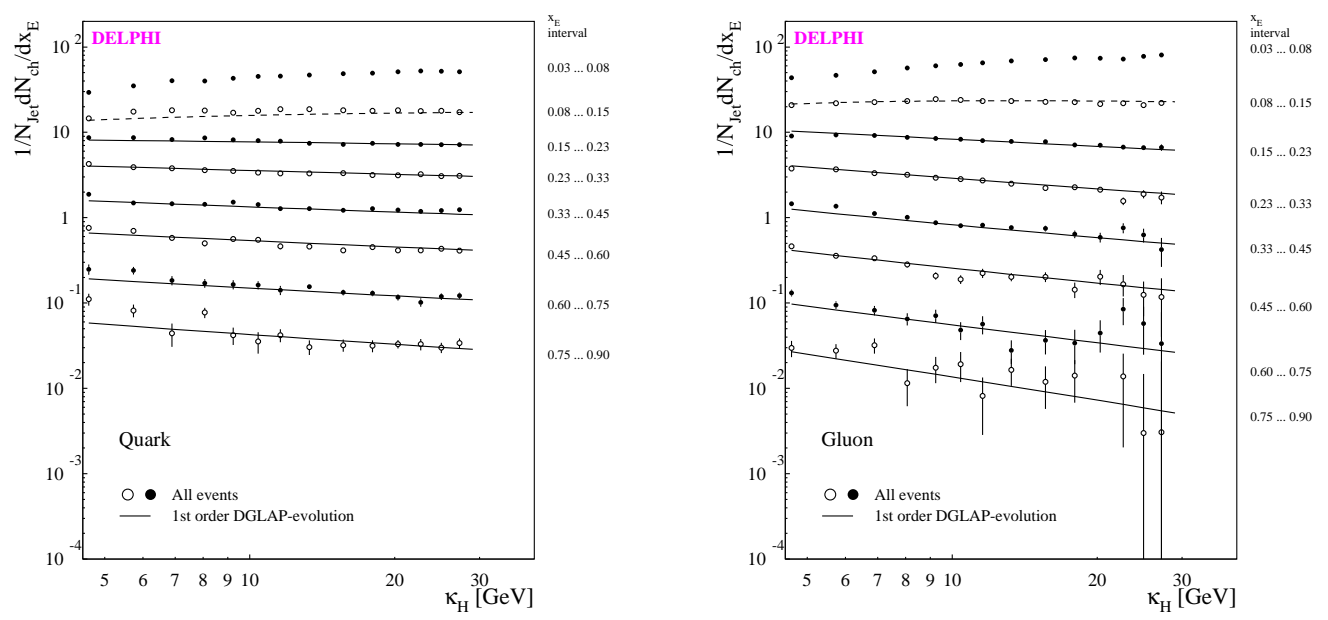

Figure 15: Scale dependence of quark and gluon fragmentation. 


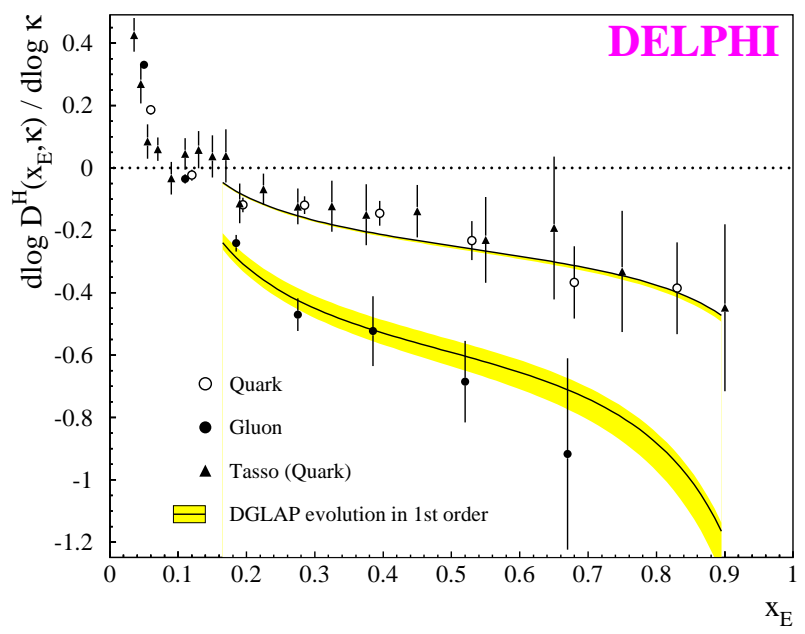

Figure 16: Logarithmic gradients of quark and gluon fragmentation.

A crucial point in the DELPHI analysis is that 3-jet events are not selected using a fixed jet resolution $y_{\text {cut }}$, but rather each event is clustered to precisely 3 jets. This avoids 'biasing' the gluon jet sample by preventing further jet emission above $y_{\text {cut }}$.

The same point is well illustrated in analyses of average multiplicities in 2- and 3 -jet events [51, 52, 53]. If $N_{q \bar{q}}(s)$ is the 'unbiased' $q \bar{q}$ multiplicity, then in events with precisely 2 jets at resolution $y_{\text {cut }}$ there a rapidity plateau of length $\ln \left(1 / y_{\text {cut }}\right)$ (see fig. 17) and the multiplicity is

$$
N_{2}\left(s, y_{\text {cut }}\right) \simeq N_{q \bar{q}}\left(s y_{\text {cut }}\right)+\ln \left(1 / y_{\text {cut }}\right) N_{q \bar{q}}^{\prime}\left(s y_{\text {cut }}\right)
$$

where $N^{\prime}(s) \equiv s d N / d s$. Clustering each event to 3 jets we get this multiplicity with $y_{3}$ in place of $y_{\text {cut }}$, plus an unbiased gluon jet:

$$
N_{3}(s) \simeq N_{2}\left(s, y_{3}\right)+\frac{1}{2} N_{g g}\left(s y_{3}\right)
$$

Thus one can extract the unbiased $g g$ multiplicity, plotted in fig. 18 vs. $p_{1}^{T} \sim \sqrt{s y_{3}}$ [54]. The ratio of $g g / q \bar{q}$ slopes gives yet another measure of $C_{A} / C_{F}$ [53]:

$$
r_{\text {mult }}=2.246 \pm 0.062 \text { (stat.) } \pm 0.080 \text { (sys.) } \pm 0.095 \text { (theo.) }
$$

\section{Current and target fragmentation in DIS}

H1 [55] and ZEUS [56] have studied the distributions of $x_{p}=2|\boldsymbol{p}| / Q$ in the current and target hemispheres in the Breit frame (fig. 19). 


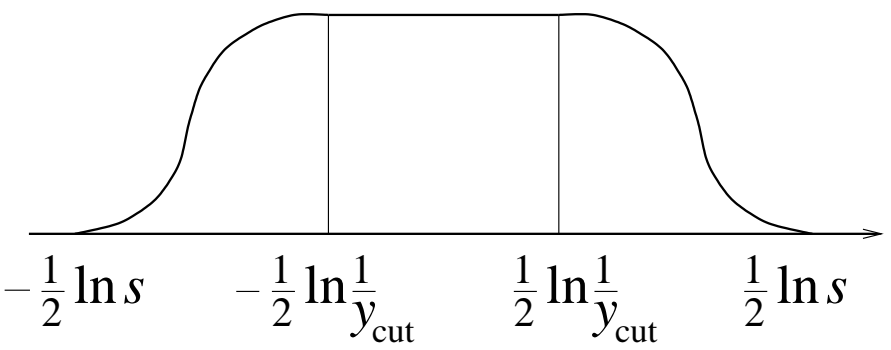

Figure 17: Rapidity plateau in two-jet events.

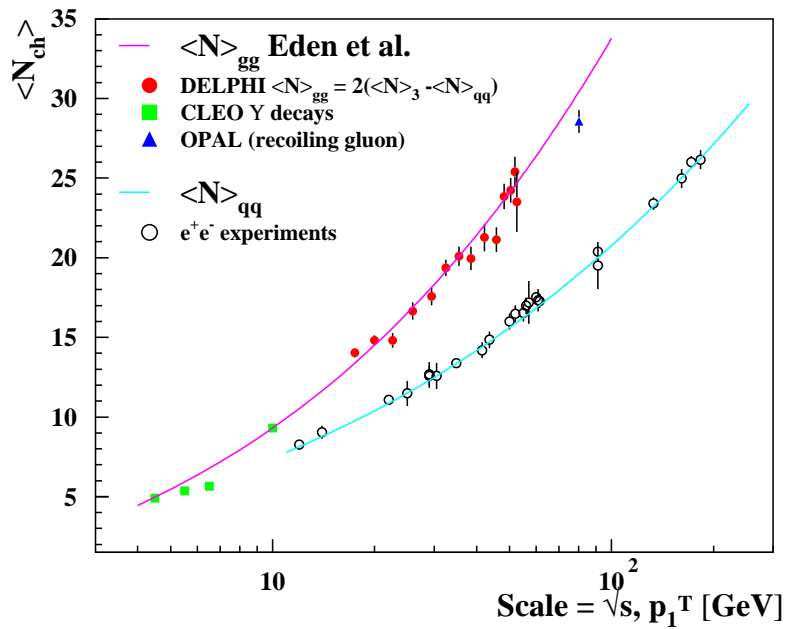

Figure 18: Average $q \bar{q}$ and $g g$ multiplicities deduced from 2- and 3-jet events.

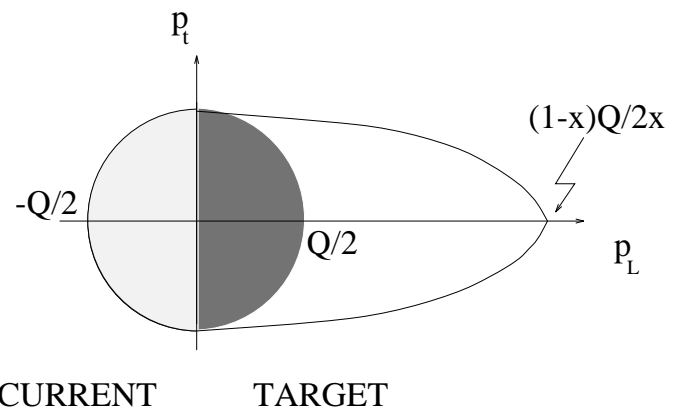

Figure 19: Breit frame current and target regions in DIS. 


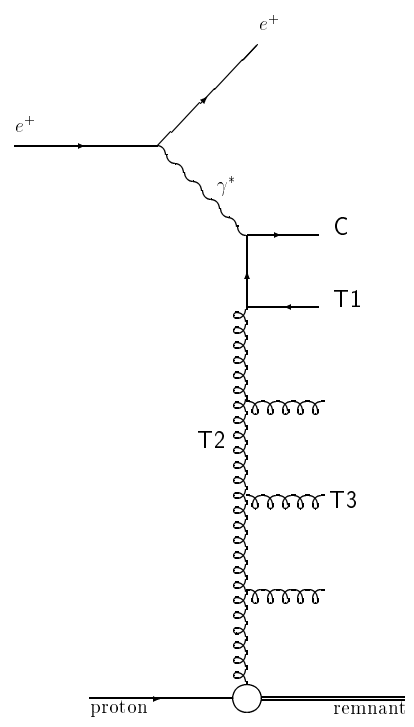

Figure 20: Contributions to the final state in DIS.

In the current hemisphere one expects fragmentation of the current jet $(\mathrm{C}$ in fig. 20), similar to half an $e^{+} e^{-}$event. In the target hemisphere, the contribution T1 is similar to C, T2 gives extra particles with $x_{p}<1$, while T3 gives $x_{p} \gtrsim 1$, generally outside detector acceptance.

- In the current hemisphere the charged multiplicity is indeed similar to $e^{+} e^{-}$ (fig. 21 [56]). Differences at low $Q^{2}$ are consistent with the expected bosongluon fusion contribution. The distribution of $\xi=\ln \left(1 / x_{p}\right)$ is also similar to $e^{+} e^{-}$, i.e. close to Gaussian with little Bjorken $x$ dependence (fig. 22).

At low $Q^{2}$ there is evidence of strong subleading corrections. The distribution is skewed towards higher values of $\xi\left(\right.$ smaller $x_{p}$ ), contrary to MLLA predictions (fig. 23). The quantity plotted is

$$
\text { Skewness } \equiv\left\langle(\xi-\bar{\xi})^{3}\right\rangle /\left\langle(\xi-\bar{\xi})^{2}\right\rangle^{\frac{3}{2}}
$$

On the other hand, the data lie well below the fixed-order perturbative prediction [57 at low $x_{p}$ and $Q^{2}$ (fig. 24). Discrepancies could be due to power-suppressed $\left(1 / Q^{2}\right)$ corrections, of dynamical and/or kinematical origin. The bands in fig. 24 correspond to an ad-hoc correction factor

$$
\left[1+\left(\frac{m_{\mathrm{eff}}}{Q x_{p}}\right)^{2}\right]^{-1} \quad\left(0.1<m_{\mathrm{eff}}<1 \mathrm{GeV}\right) .
$$




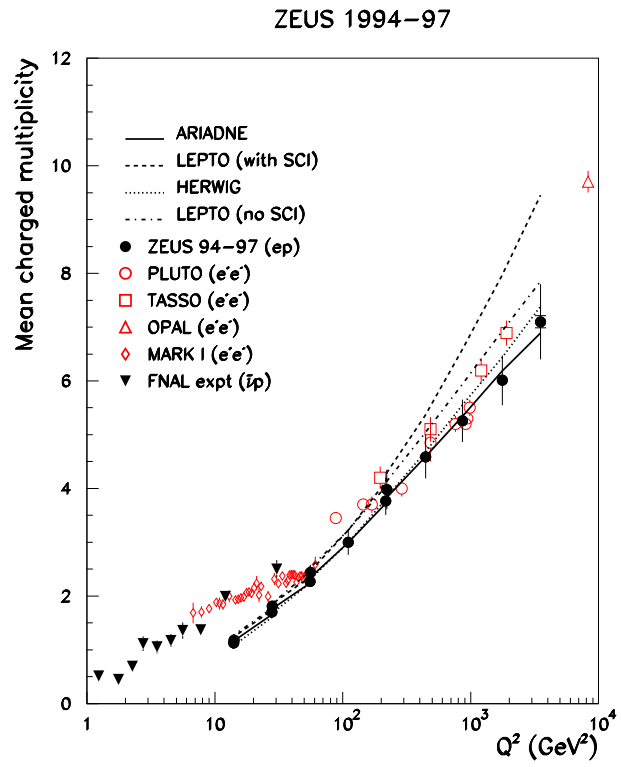

Figure 21: Charged multiplicity in current hemisphere.

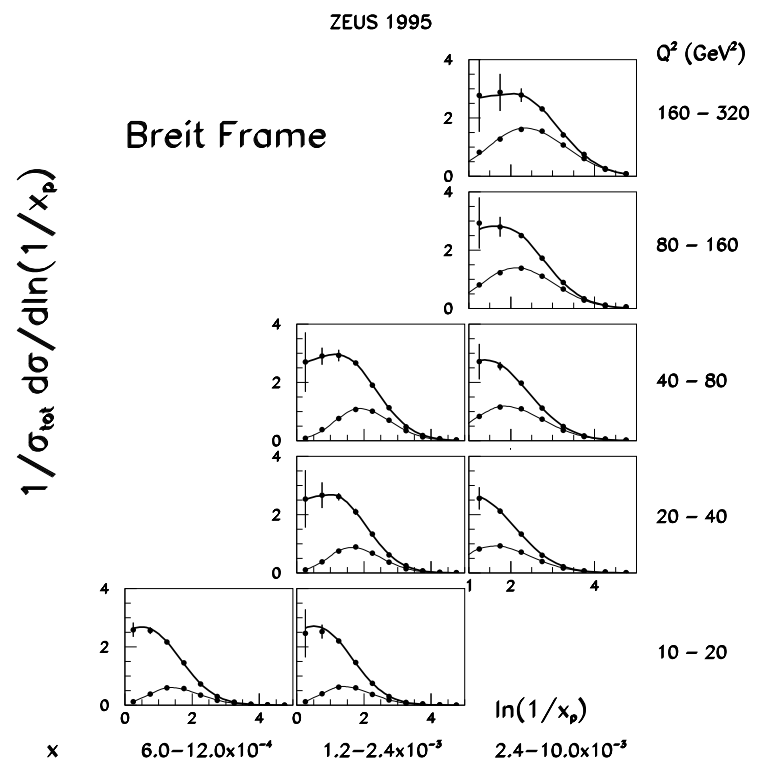

Figure 22: Fragmentation in DIS. Upper data (heavy curve) target region, lower data (light curve) current region. 


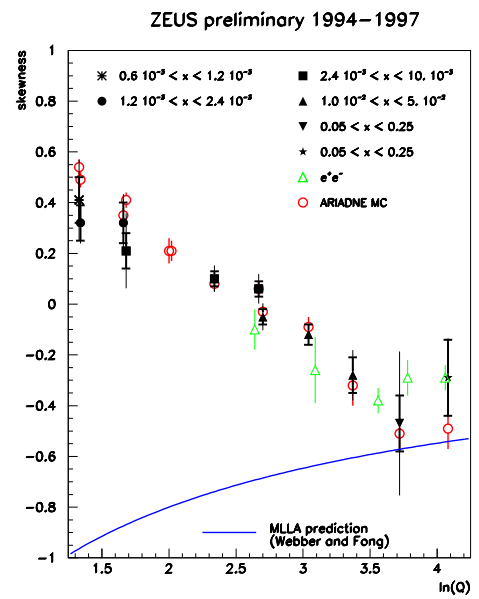

Figure 23: Skewness in current fragmentation region.

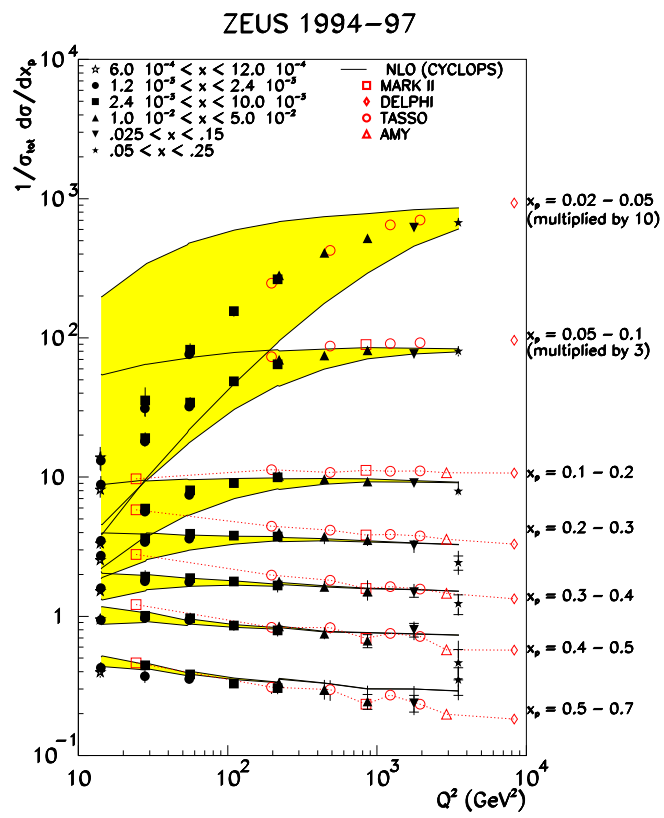

Figure 24: Scaling violation in DIS fragmentation. 


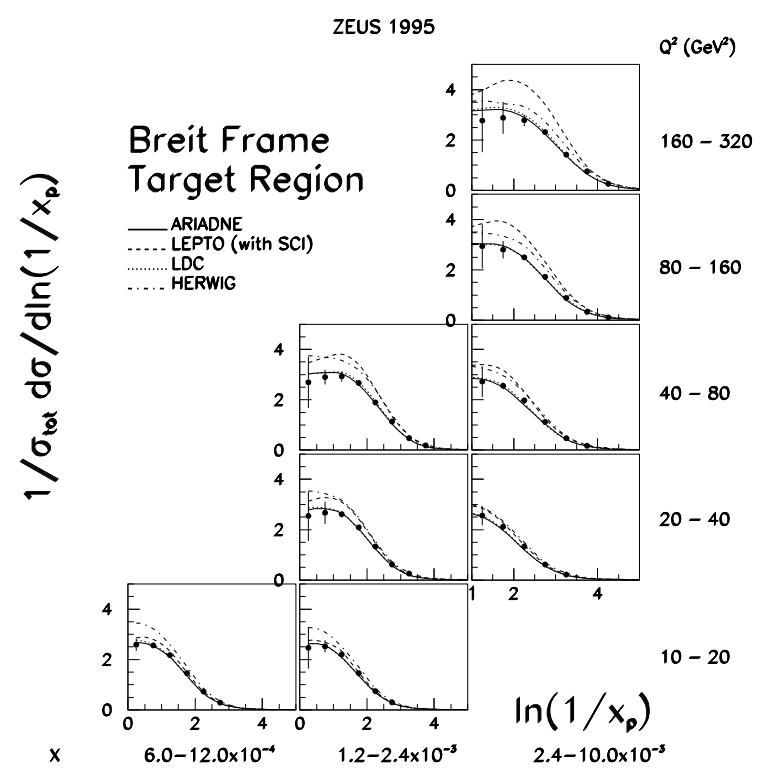

Figure 25: Target fragmentation compared with models.

- In the target hemisphere there is also disagreement with MLLA [56], possibly due to the T3 contribution "leaking" into the region $x_{p}<1$. If anything, Monte Carlo models predict too much leakage (fig. 25). Little $Q^{2}$ dependence is evident.

\section{Heavy quark fragmentation}

New data on $b \rightarrow \mathrm{B}$ fragmentation from SLD [58], using high-precision vertexing, discriminate between parton-shower plus hadronization models (fig. 26). Note that the data have not yet been corrected for detector effects.

Including more perturbative QCD leads to a reduction in the amount of nonperturbative smearing required to fit the data. Non-perturbative effects are conventionally parametrized by $\epsilon_{b}$ in the Peterson function [59]

$$
f(z)=\frac{1}{z}\left(1-\frac{1}{z}-\frac{\epsilon_{b}}{1-z}\right)^{-2} \quad\left(z=x_{B} / x_{b}\right)
$$

Pure Peterson [58: $\epsilon_{b}=0.036$.

JETSET $\left(\simeq\right.$ LLA QCD) + Peterson [58]: $\epsilon_{b}=0.006$.

NLLA QCD + Peterson [60]: $\epsilon_{b}=0.002$ (fig. 27). 


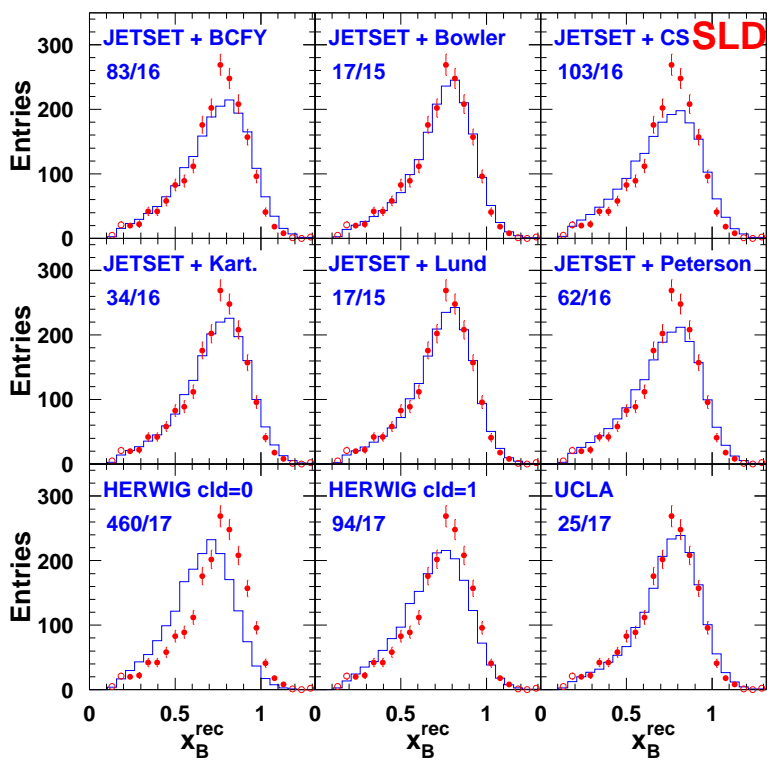

Figure 26: SLD data on $b \rightarrow \mathrm{B}$ fragmentation compared with models.
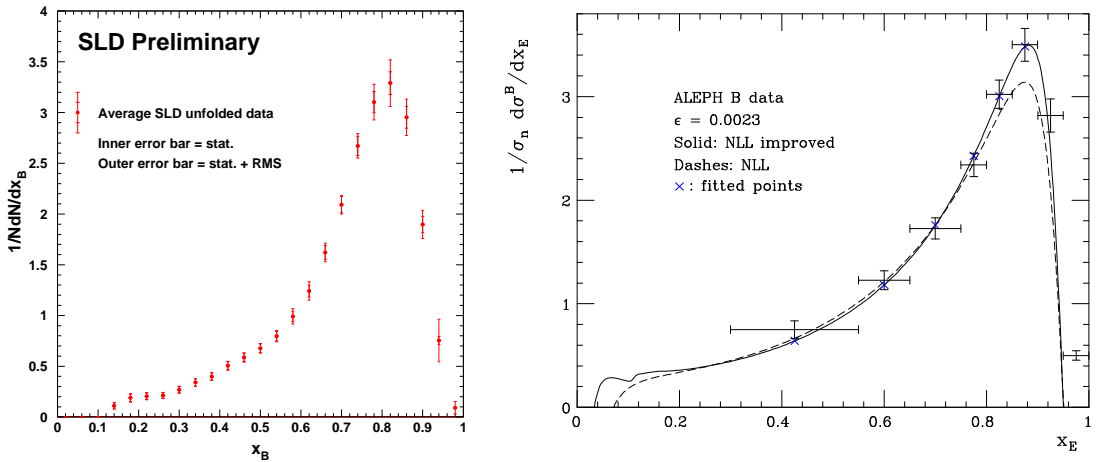

Figure 27: SLD [58 and ALEPH [61] data on $b \rightarrow \mathrm{B}$ fragmentation, the latter compared with NLLA QCD 60]. 

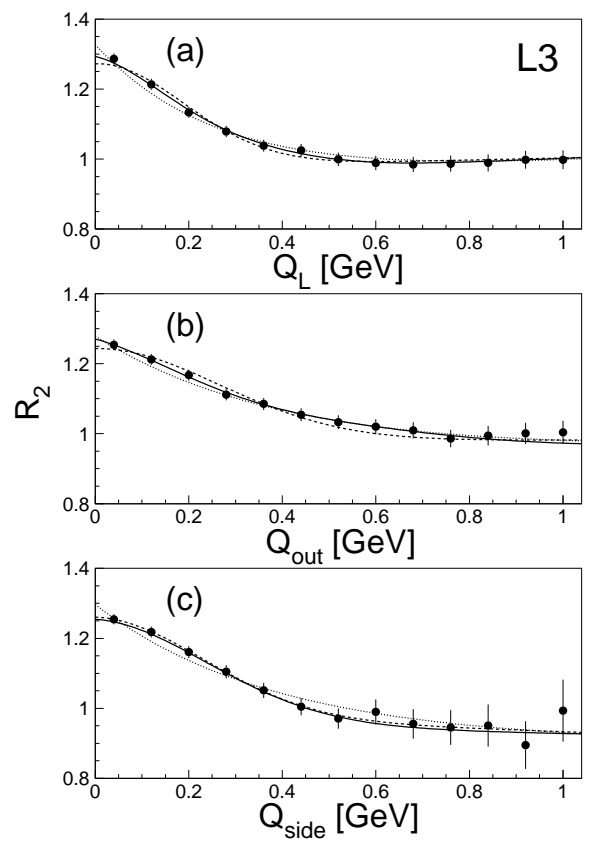

Figure 28: Bose-Einstein correlations with repect to axes along and perpendicular to the thrust axis.

In the universal low-scale $\alpha_{\mathrm{S}}$ model, the perturbative prediction is extrapolated smoothly to the non-perturbative region, with no Peterson function at all [20].

\section{Bose-Einstein correlations}

Studies of $\pi^{ \pm} \pi^{ \pm}$correlations which distinguish between directions along and perpendicular to the thrust axis find definite evidence for elongation of the source region along that axis (fig. 28 and table 3 [62, 63, 64]). This has a good explanation in the Lund string model, in terms of the change of the space-time area $A$ in fig. t when identical bosons are interchanged [65].

ALEPH [66] has clear evidence of Fermi-Dirac anticorrelation in $\Lambda \Lambda(\mathrm{S}=1)$. Plots $\mathrm{A}-\mathrm{C}$ in fig. 29 correspond to different comparison (no-correlation) samples. The source size appears to decrease with increasing particle mass (table 4 [66, 67, 68]). However, some of this effect is kinematic 69. 


\begin{tabular}{|c|c|c|c|}
\hline Expt & $R_{L}(\mathrm{fm})$ & $R_{T}(\mathrm{fm})$ & $R_{L} / R_{T}$ \\
\hline DELPHI & $0.85 \pm 0.04$ & $0.53 \pm 0.04$ & $1.61 \pm 0.10$ \\
L3 & $0.74 \pm 0.04$ & $0.56_{-0.06}^{+0.03}$ & $1.23 \pm 0.03_{-0.13}^{+0.40}$ \\
OPAL & $0.935 \pm 0.029$ & $0.720 \pm 0.045$ & $1.30 \pm 0.12$ \\
\hline
\end{tabular}

Table 3: Longitudinal and transverse source radii.

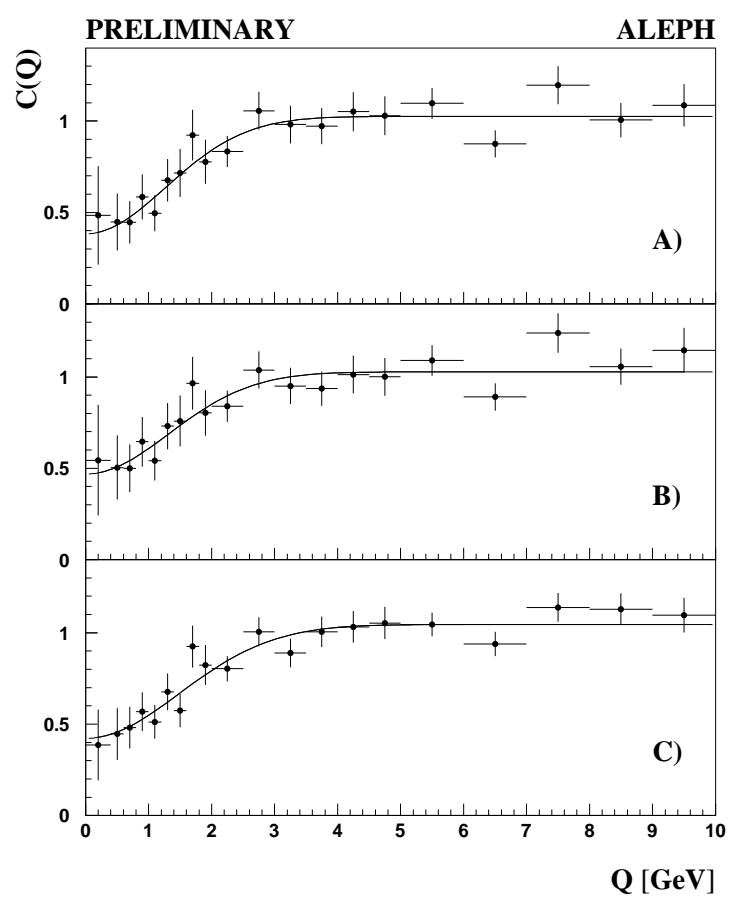

Figure 29: Fermi-Dirac correlation for $\Lambda \Lambda$. 


\begin{tabular}{|c|c|}
\hline Particles & $R_{\text {source }}(\mathrm{fm})$ \\
\hline$\pi \pi$ & $0.65 \pm 0.04 \pm 0.16$ \\
$\mathrm{KK}$ & $0.48 \pm 0.04 \pm 0.07$ \\
$\Lambda \Lambda$ & $0.11 \pm 0.02 \pm 0.01$ \\
\hline
\end{tabular}

Table 4: Comparison of source radii.

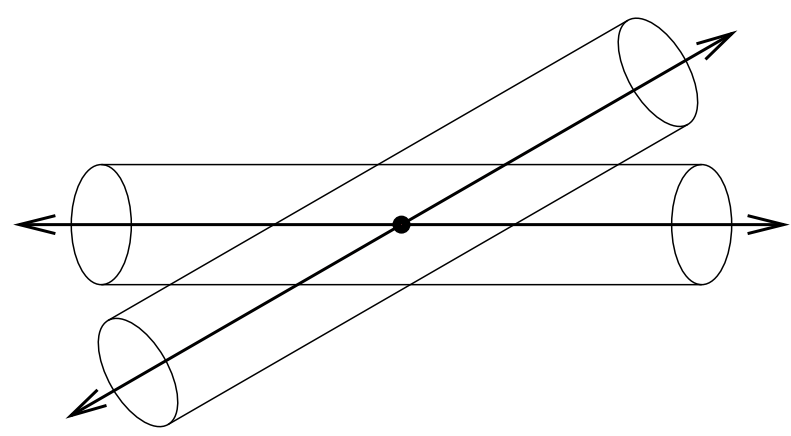

Figure 30: Hadronizations volumes in WW decay.

\section{WW fragmentation}

In $e^{+} e^{-} \rightarrow \mathrm{WW}$, we would expect correlations between $\mathrm{W}$ hadronic decays due to overlap of hadronization volumes. This occurs mainly in the central region, and is orientation-dependent (fig. 30). These reconnection effects have been searched for in single-particle distributions. One would expect discrepancies between the distributions in semi-leptonic and fully hadronic decays, especially at low momenta. There is no firm evidence yet for such effects in the $x_{p}$ distribution (fig. 31 [70]). However, DELPHI [42] report a possible small $\left(\sim 2 \sigma\right.$ ?) effect in the distribution of $p_{T}$ relative to the thrust axis (fig. 32).

Bose-Einstein correlations between hadrons from different W's are also being looked for. They would lead to an increase in the correlation function for WW relative to that for a single $\mathrm{W}$. There is no sign of any increase at present (fig. 33 [71]).

\section{Summary}

- Detailed fragmentation studies need more theoretical input in the form of coefficient functions that take account of selection procedures, especially for gluon 


\section{ALEPH PRELIMINARY 189GeV}
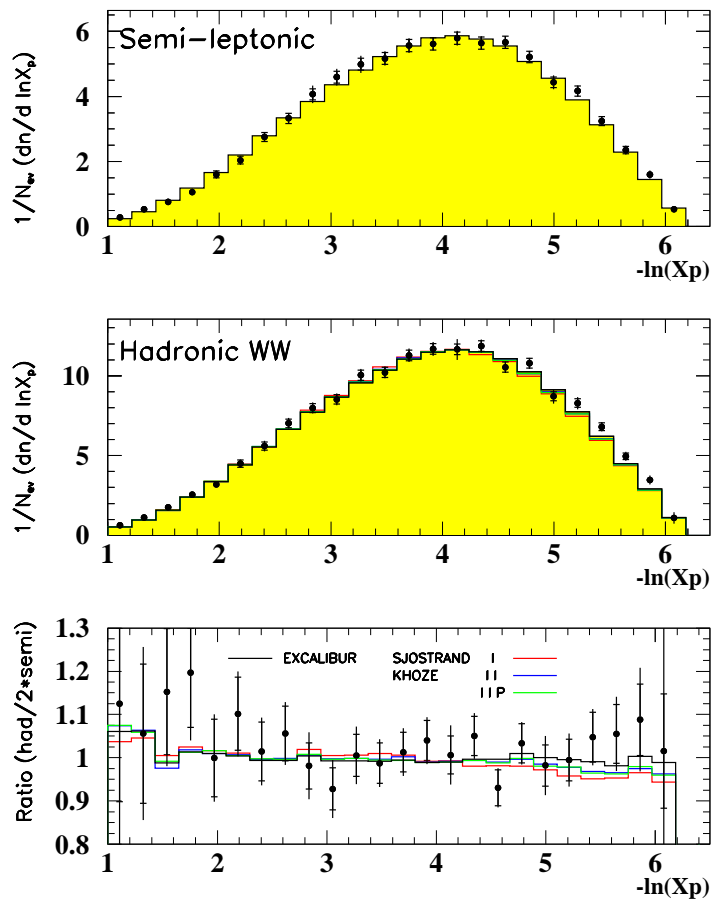

Figure 31: Distribution of momentum fraction in WW decay. 

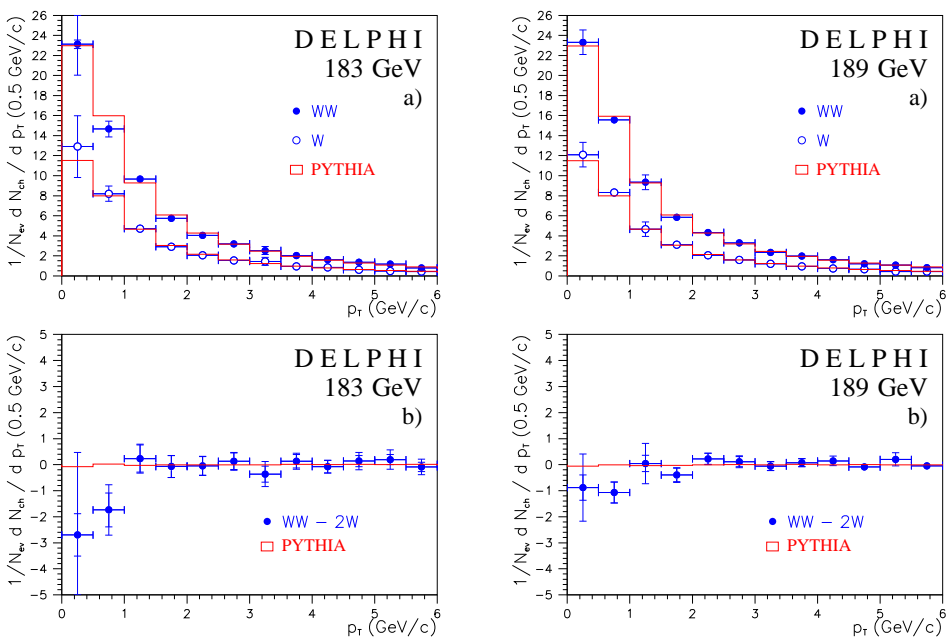

Figure 32: Distribution of transverse momentum in WW decay.

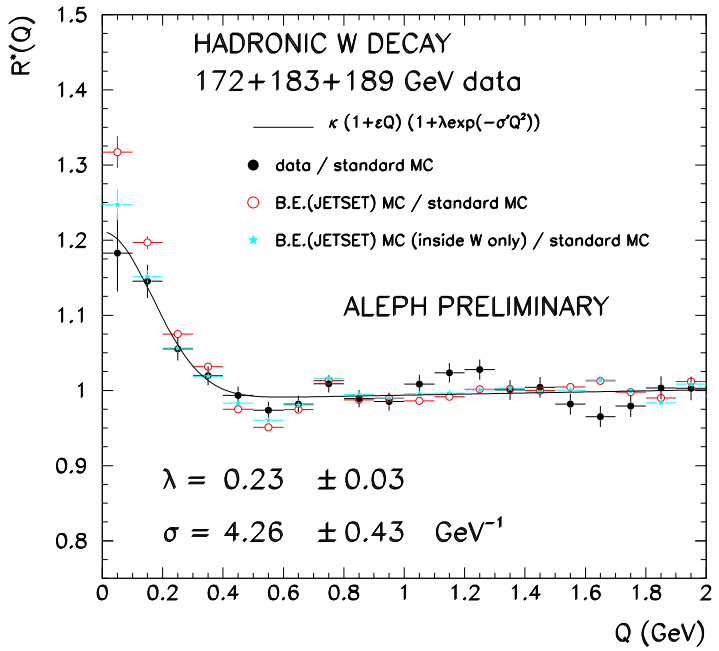

Figure 33: Bose-Einstein correlations in WW decay. 
jets in $e^{+} e^{-}$final states.

- Hadronization studies suggest that particle masses, rather than quantum numbers, are the dominant factor in suppressing heavy particle production. Baryon production is not yet well described by any model.

- Quark and gluon jets have the expected differences and these can be used to measure the ratio of colour factors $C_{A} / C_{F}$. There is no strong evidence yet for different particle content in gluon jets.

- Fragmentation in DIS shows disagreements with perturbative predictions. It is not yet clear whether these are due to higher-order or non-perturbative effects.

- New precise $b$ quark fragmentation data test models and suggest that perturbative effects dominate.

- Bose-Einstein (Fermi-Dirac) correlations show elongation of the source along the jet axis and source shrinkage with increasing mass.

- WW fragmentation still shows no firm evidence for correlation between the decay products of the two W's.

\section{References}

[1] J. Womersley, these proceedings.

[2] M.L. Mangano, plenary talk at 1999 EPS Conference, Tampere, Finland, July, 1999, hep-ph/9911256.

[3] J.C. Collins and D.E. Soper, Ann. Rev. Nucl. Part. Sci. 37, 383 (1987).

[4] See the fragmentation web pages of the European network "Hadronic Physics with High Energy Electromagnetic Probes" (HaPHEEP) for much useful information on this topic:

http://droide1.pv.infn.it/FFdatabase/fragmentation.html

[5] M. Beneke, these proceedings.

[6] http://wwW.physics.helsinki.fi/〜hep99//

[7] Aleph Collaboration, http://alephwww.cern.ch/ALPUB/conf/conf.html

[8] DELPHI Collaboration, http://delphiwww.cern.ch/〜pubxx/delwww/www/ delsec/conferences/tampere99/ 
[9] L3 Collaboration, http://13www.cern.ch/conferences/EPS99/

[10] OPAL Collaboration, http://www.cern.ch/Opal/pubs/eps99_sub.htm]

[11] SLD Collaboration, http://www-pnp.physics.ox.ac.uk/ burrows/tampere/

[12] H1 Collaboration, http://www-h1.desy.de/h1/www/publications/conf/list.tampere99.html

[13] ZEUS Collaboration, http://zedy00.desy.de/conferences99/

[14] D $\emptyset$ Collaboration, http://www-d0.fnal.gov/〜ellison/eps99/eps99.html

[15] Y.I. Azimov, Y.L. Dokshitzer, V.A. Khoze and S.I. Troian, Zeit. Phys. C31, 213 (1986).

[16] C.P. Fong and B.R. Webber, Phys. Lett. B229, 289 (1989); Nucl. Phys. B355, 54 (1991).

[17] Y.L. Dokshitzer, V.A. Khoze, A.H. Mueller and S.I. Troian, "Basics of perturbative QCD," Gif-sur-Yvette, France: Ed. Frontieres (1991) 274 p. (Basics of).

[18] Y.I. Azimov, Y.L. Dokshitzer, V.A. Khoze and S.I. Troian, Z. Phys. C27, 65 (1985).

[19] Y.L. Dokshitzer and B.R. Webber, Phys. Lett. B352, 451 (1995) hepph/9504219.

[20] Y.L. Dokshitzer, V.A. Khoze and S.I. Troian, Phys. Rev. D53, 89 (1996) hepph/9506425.

[21] Y.L. Dokshitzer, G. Marchesini and B.R. Webber, Nucl. Phys. B469, 93 (1996) hep-ph/9512336.

[22] G. Marchesini and B.R. Webber, Nucl. Phys. B238, 1 (1984).

[23] B.R. Webber, Nucl. Phys. B238, 492 (1984).

[24] G. Marchesini and B.R. Webber, Nucl. Phys. B310, 461 (1988).

[25] G. Marchesini, B.R. Webber, G. Abbiendi, I.G. Knowles, M.H. Seymour and L. Stanco, Comput. Phys. Commun. 67, 465 (1992).

[26] G. Marchesini, B.R. Webber, G. Abbiendi, I.G. Knowles, M.H. Seymour and L. Stanco, hep-ph/9607393.

[27] D. Amati and G. Veneziano, Phys. Lett. 83B, 87 (1979). 
[28] G. Marchesini, L. Trentadue and G. Veneziano, Nucl. Phys. B181, 335 (1981).

[29] I.G. Knowles and G.D. Lafferty, J. Phys. G G23, 731 (1997) hep-ph/9705217.

[30] B. Andersson, G. Gustafson, G. Ingelman and T. Sjostrand, Phys. Rept. 97, 31 (1983).

[31] T. Sjostrand, Comput. Phys. Commun. 39, 347 (1986).

[32] T. Sjostrand and M. Bengtsson, Comput. Phys. Commun. 43, 367 (1987).

[33] T. Sjostrand, Comput. Phys. Commun. 82, 74 (1994).

[34] T. Sjostrand, CERN-TH-7112-93, hep-ph/9508391.

[35] C.D. Buchanan and S.B. Chun, Phys. Rev. Lett. 59, 1997 (1987).

[36] S.B. Chun and C.D. Buchanan, Phys. Lett. B308, 153 (1993).

[37] S. Chun and C. Buchanan, Phys. Rept. 292, 239 (1998).

[38] G. Rudolph, private communication.

[39] P.V. Chliapnikov, Phys. Lett. B462, 341 (1999).

[40] G. Alexander et al. [OPAL Collaboration], Z. Phys. C73, 569 (1997).

[41] DELPHI Collaboration, EPS-HEP99 paper 3_147.

[42] DELPHI Collaboration, EPS-HEP99 paper 1_225.

[43] DELPHI Collaboration, EPS-HEP99 paper 1_225.

[44] CDF Collaboration, EPS-HEP99 paper 1_600.

[45] K. Abe et al. [SLD Collaboration], hep-ex/9908033.

[46] DELPHI Collaboration, EPS-HEP99 paper 3_146.

[47] OPAL Collaboration, EPS-HEP99 paper 1_4.

[48] G. Abbiendi et al. [OPAL Collaboration], Eur. Phys. J. C11, 217 (1999) hepex/9903027.

[49] DELPHI Collaboration, EPS-HEP99 paper 1_571.

[50] S. Catani, Y.L. Dokshitzer, M. Olsson, G. Turnock and B.R. Webber, Phys. Lett. B269, 432 (1991). 
[51] S. Catani, B.R. Webber, Y.L. Dokshitzer and F. Fiorani, Nucl. Phys. B383, 419 (1992).

[52] P. Eden, G. Gustafson and V. Khoze, Eur. Phys. J. C11, 345 (1999) hepph/9904455.

[53] P. Abreu et al. [DELPHI Collaboration], Phys. Lett. B449, 383 (1999) hepex/9903073.

[54] DELPHI Collaboration, private communication.

[55] C. Adloff et al. [H1 Collaboration], Nucl. Phys. B504 (1997) 3 hep-ex/9707005.

[56] J. Breitweg et al. [ZEUS Collaboration], Eur. Phys. J. C11, 251 (1999) hepex/9903056.

[57] D. Graudenz, Phys. Lett. B406, 178 (1997) hep-ph/9606470.

[58] K. Abe et al. [SLD Collaboration], hep-ex/9908032.

[59] C. Peterson, D. Schlatter, I. Schmitt and P. Zerwas, Phys. Rev. D27, 105 (1983).

[60] P. Nason and C. Oleari, hep-ph/9903541.

[61] D. Buskulic et al. [ALEPH Collaboration], Phys. Lett. B357, 699 (1995).

[62] DELPHI Collaboration, EPS-HEP99 paper 1_221.

[63] L3 Collaboration, EPS-HEP99 paper 3_280.

[64] OPAL Collaboration, EPS-HEP99 paper 3_64.

[65] B. Andersson and M. Ringner, Phys. Lett. B421, 283 (1998) hep-ph/9710334.

[66] ALEPH Collaboration, EPS-HEP99 paper 1_389.

[67] D. Decamp et al. [ALEPH Collaboration], Z. Phys. C54, 75 (1992).

[68] P. Abreu et al. [DELPHI Collaboration], Phys. Lett. B379, 330 (1996).

[69] M. Smith, hep-ph/9912250.

[70] ALEPH Collaboration, EPS-HEP99 paper 1_387.

[71] ALEPH Collaboration, EPS-HEP99 paper 1_388. 


\section{Discussion}

Charles Buchanan (UCLA): To elaborate on the UCLA approach to hadronization: We find that the idea of a spacetime area law (as suggested by strong QCD) works very well as an organizing principle in the stage of the soft strong-coupled hadronization part of the process - that is, it gracefully predicts "easy" data such as light-quark meson production rates and distributions in $e^{+} e^{-}$with few parameters and forms an attractive basis for studying the relation with the perturbative stage and more complicated phenomena such as baryon formation and $p_{T}$ effects. To study these latter in detail, we have joined BaBar where we will use the $10^{8}$ high quality events to be collected in the next 2-3 years to study for baryon-meson-antibaryon 3 -body corrections, $p_{T}$ correlations, etc. We invite physicists interested in the area to contact us (at buchanan@physics.ucla.edu).

George Hou (National Taiwan University): Regarding particle content of gluon jets, is there any result on the $\eta^{\prime}$ content? This particle is more naturally associated with gluons than $\eta$ or $\phi$.

Webber: As far as I know, there are no results available yet on the $\eta^{\prime}$ content of gluon jets.

Michael Peskin (SLAC): You have shown that, when quark and gluon jets are carefully selected, their average properties are clearly distinguished. Of course, what one really wants is a variable which allows one to separate quark and gluon jets (if only statistically) in the Tevatron or LHC environment. What is the best choice for this purpose?

Webber: This is difficult to do because, although the average properties are different, the fluctuations are large.

Tom Ferbel (University of Rochester): Actually, D0 has used the differences between quark and gluon jets very effectively, in a statistical manner (with neural

networks) to improve the signal to background in the analysis of $t \bar{t}$ production in the all-jets channel. 